\title{
EVALUATION OF QUANTITATIVE CRP IN NEONATAL SEPSIS DIAGNOSIS
}

M. Fatemi Khorasgani, L. Moaied Alaee, F. Pajang

Pediatrics, Islamic Azad University- Najafabad Branch-Shariati Hospital, Isfahan, Iran

Introduction: Neonatal sepsis diagnosis is a problem, so pediatricians use laboratory direct methods (blood, urine, CSF culture...) and indirect methods( leukocyte count, ESR, qualitative and quantitative CRP ...) for better evaluation. This study is about quantitative CRP in sepsis .

Material and methods: It is a cross sectional study in neonatal ward of Shariati Hospital in Isfahan in September 2008, January and February 2009.50 neonates with sepsis clinical diagnosis selected. Quantitative CRP measured at first 6 hours of diagnosis and 12-48 hours later along other laboratory exams. CRP positive range is $>8 \mathrm{mg} \%$. Neonates divided in 4 groups: 1 - Positive blood culture( absolute diagnosis) :6(12\%) 2Clinical diagnosis and at least one positive indirect exam( Full clinical sepsis) : 22(44\%) 3-Clinical diagnosis without positive indirect exam( Probable sepsis) : 4(8\%) and 4-Neonates who had another diagnosis during admission : $18(36 \%)$.

Results: Quantitative CRP was negative in 36 patients which 18 were in fourth group, 16 in second and 2 in first. In 14 patients the exam was positive which 4 were in first, 6 in second and 4 were in third group. Second CRP measured in 36 patients which from 30 negatives 20 were in fourth, 2 in third, 14 in second and 4 in first group and from 6 positive 4 in second and 2 were in first group. Upon analytic methods, Positive predictor value for CRP>8 is $100 \%$ and Negative predictor value is $50 \%$.

Conclusion: Quantitative CRP measurement is a useful predictor method in neonatal sepsis diagnosis. 